\title{
Wissenschaftliches Kolloquium
}

Entwicklung eines rauscharmen Lagemesssystems für sensorlose magnetisch gelagerte Antriebe mit FlussBypass-Kompensation für hohe Sättigungstoleranz

\author{
M.Sc. André Schleicher \\ Technische Universität Chemnitz \\ Fakultät für Elektrotechnik und Informationstechnik \\ Professur Elektrische Energiewandlungssysteme und Antriebe
}




\section{Motivation}

Sog. „sensorloser“ Antrieb:

- keine Lagesensoren nötig, Rotorlageermittlung erfolgt über Messung elektrischer und magnetischer Größen des Motors

- Fertigungsaufwand und Kosten ggf. reduziert

- einfacher und robuster Aufbau des Motors

- im Gegensatz zu anderen phys. Messprinzipien Unempfindlichkeit gegenüber elektrischen Feldern, Verschmutzungen, Feuchtigkeit, Licht, etc.

- Lagemessung erfolgt direkt von Stator zu Rotor - keine Messspuren, kein temperaturabhängiger Versatz zwischen Sensor und Blechpaket 


\section{Untersuchungsschwerpunkte}

Anforderungen an sensorloses Lagemesssystem:

- Grenzfrequenz $f_{G}>10 \mathrm{kHz}$ (für Magnetlagerungen und lagerlose Motoren)

- hoher Signal-Rauschabstand

- funktionsfähig bis hin zu möglichst hohem mag. Fluss (Sättigung), für hohe magnetische Ausnutzung des Motors 


\section{Theoretische Grundlagen}

1. Magnetischer Fluss: $\quad \Phi=\Phi(\delta(t), I(t), t)$

gesucht: (magnetische)

Luftspaltlänge

Wicklungsstrom

2. Indirekte Messung von $\delta$ über Messung von $\Phi$ :

a) über Flussdichte $\boldsymbol{B}$-> Hallelemente

b) über $U$ und $I$ mittels Induktionsgesetz:

$$
U=R I+\frac{\mathrm{d} \Phi}{\mathrm{d} t}=R I+L_{d} \dot{I}+\frac{\partial \Phi}{\partial \delta} \dot{\delta}+\frac{\partial \Phi}{\partial t}
$$

diff. Induktivität $L_{d}=\frac{\partial \Phi}{\partial I}$ (Anstieg Magnetisierungskurve)
Geometrieänderung expl. Zeitabhängigkeit (Wirbelströme, etc.) 


\section{Stand der Technik}

\section{1. „Direct Digital Inductance Estimation“ (DDIE) [1], [2]:}

- Messung von Strom I (über Shunt oder Stromwandler) und ggf. Spannung der Hauptwicklung $U$, zeitlich hoch aufgelöst

- Schätzung der Größen $R$ und $L_{d}$ sowie ggf. Restterm $\frac{\partial \Phi}{\partial \delta} \dot{\delta}+\frac{\partial \Phi}{\partial t}$ aus Zeitverlauf von $I$

- Vorteile:

- einfachster Messaufbau

- Nachteile:

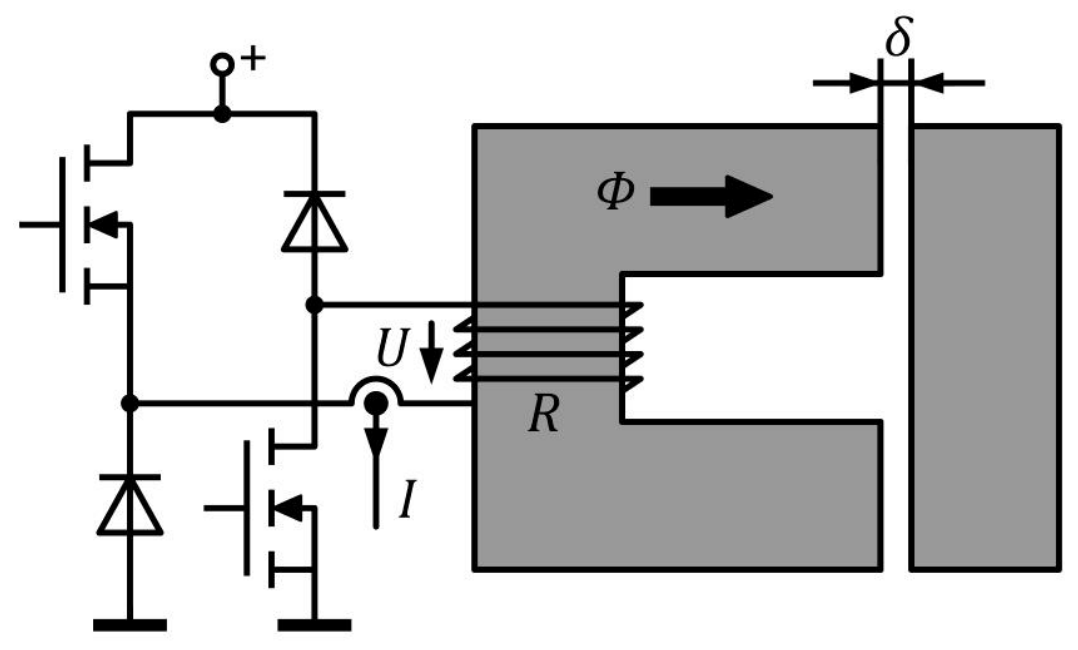

- großes Rauschen durch Schätzung von $I$ sowie kleine Messspannung (Shunt bzw. Hallelement in Stromwandler)

- $\quad$ bei Verwendung rauscharmer Stromwandler (z.B. Nullflusswandler) teuer

- $\quad$ keine Kompensation des Sättigungseinflusses, nur geringer Fluss tolerierbar

- Hauptfluss folgt Strom zeitverzögert

- $\quad$ sehr hohe Rechenleistung nötig 


\section{Stand der Technik}

\section{Streuflussmessung [3]:}

- Gesamtfluss $\Phi_{G}$ teilt sich lageabhängig auf in Hauptfluss $\Phi_{H}$ und Streufluss $\Phi_{\sigma}$

- Messung von Wicklungsstrom und Streuflussdichte $B_{\sigma}$ mittels Hallelement

- Vorteile:

- einfacher, kostengünstiger Aufbau

- $\quad$ einfache Signalauswertung

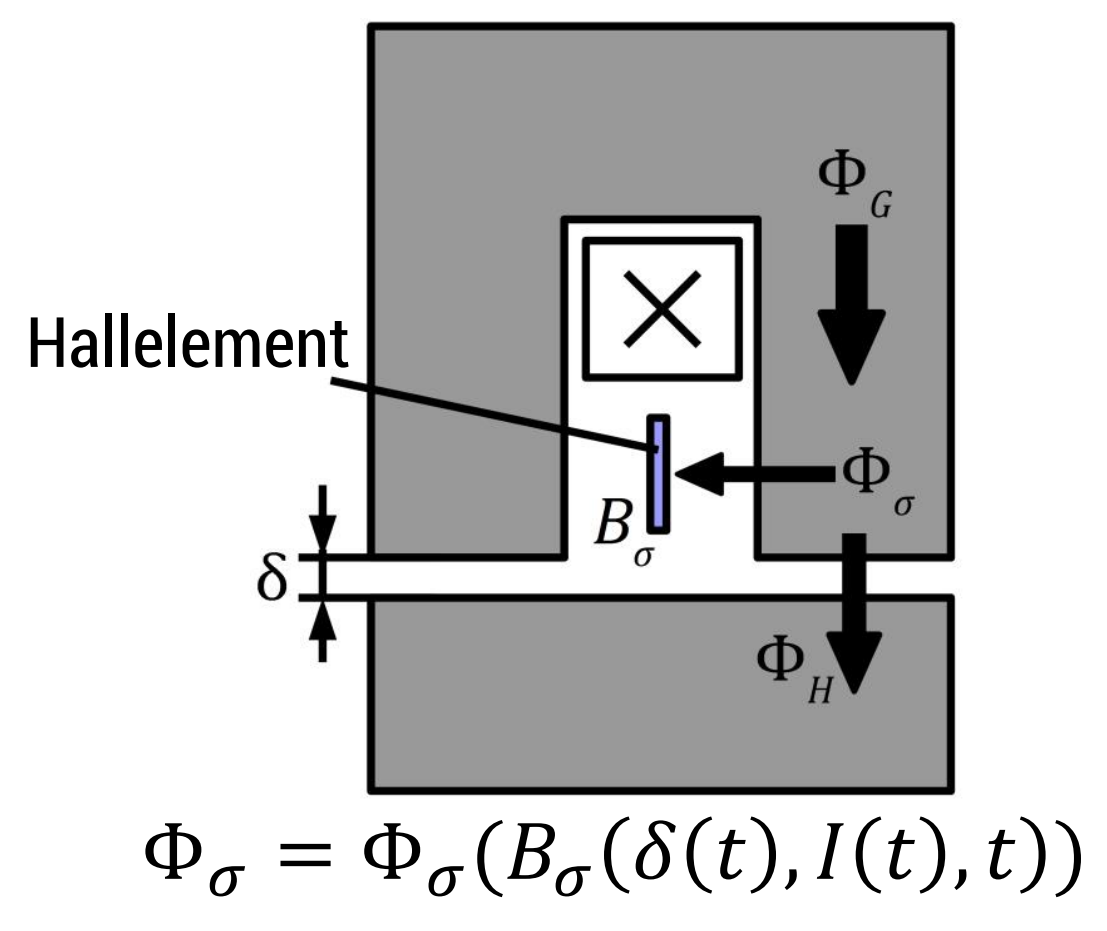

- galvanische Trennung von Messsignal und Hauptwicklung

- funktioniert auch ohne geschaltete Spannung

- Nachteile:

- großes Rauschen durch Hall-Elemente

- $\quad$ keine Kompensation des Sättigungseinflusses, moderater Fluss tolerierbar

- $\quad$ Streufluss $\Phi_{\sigma}$ folgt Strom zeitverzögert 


\section{Fluss-Bypass-Verfahren}

\section{Neuartiges Fluss-Bypass-Verfahren:}

- Erweiterung des Magnetkreises um kleinen Fluss-Bypass aus gleichem Material

- Messung von Strom I sowie der von Gesamtfluss $\Phi_{G}$ und Bypassfluss $\Phi_{B}$ induzierten Spannungen $U_{G}$ und $U_{B}$

- digitale Berechnung des Nutzsignals $Q=\frac{U_{B}-U_{G}}{U_{G}}=\frac{N_{B} \dot{\Phi}_{B}-N_{G} \dot{\Phi}_{G}}{N_{G} \dot{\Phi}_{G}}$
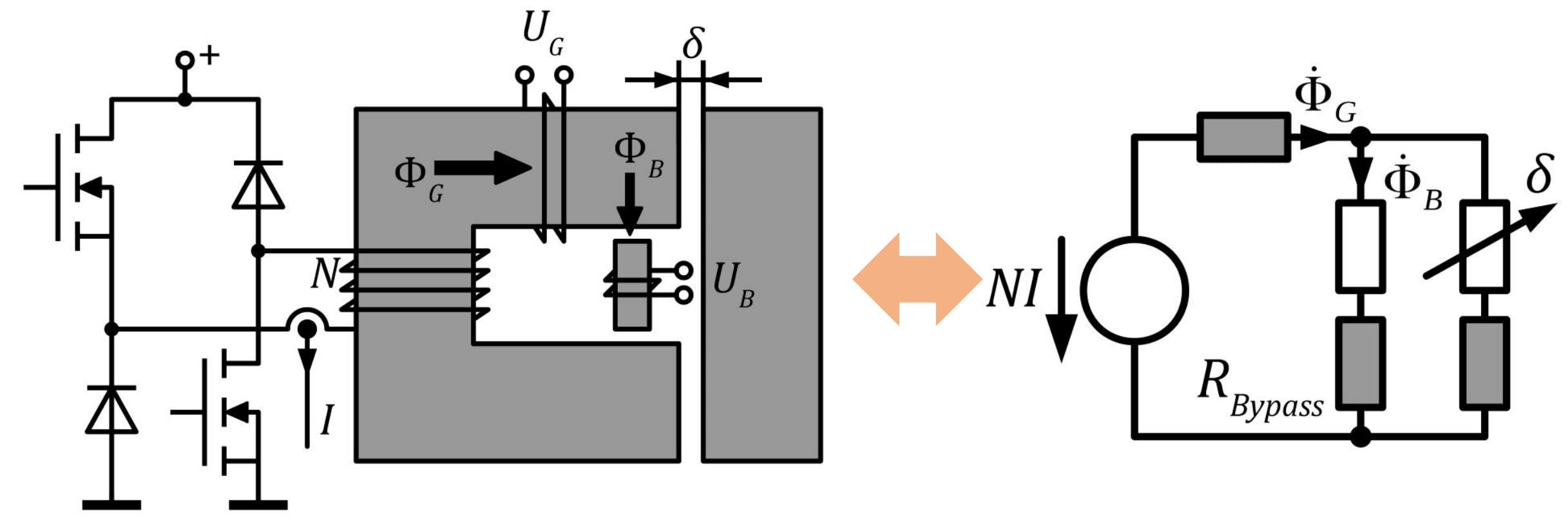


\section{Fluss-Bypass-Verfahren}

- Vorteile:

- Kompensation der Sättigung, da Fluss-Bypass und restlicher Magnetkreis aus gleichem Material

- einfache Signalauswertung: Division zweier Rohsignale

- galvanische Trennung von Messsignalen und Hauptwicklung

- sehr geringes Rauschen erreichbar aufgrund hoher Signalspannung

- Nachteile:

- Fluss-Bypass erhöht Streuinduktivität bzw. Blindleistungsbedarf des Motors

- regelmäßige Wicklungsspannungspulse mit Mindestlänge (hier: ca. $2 \mu \mathrm{s}$ ) für Messung nötig 


\section{Schema Versuchsaufbau}

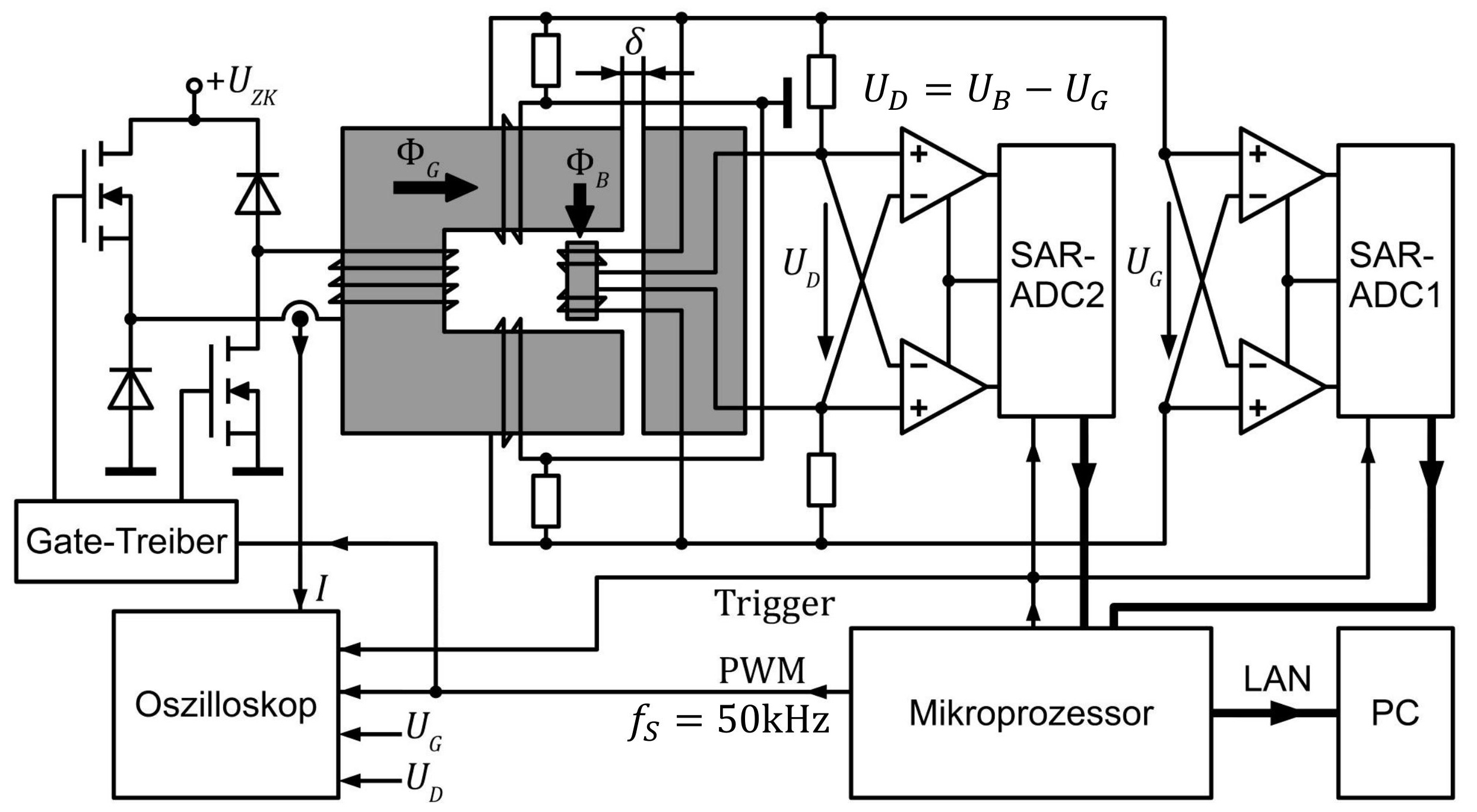




\section{醉 \\ Fotos Versuchsaufbau}

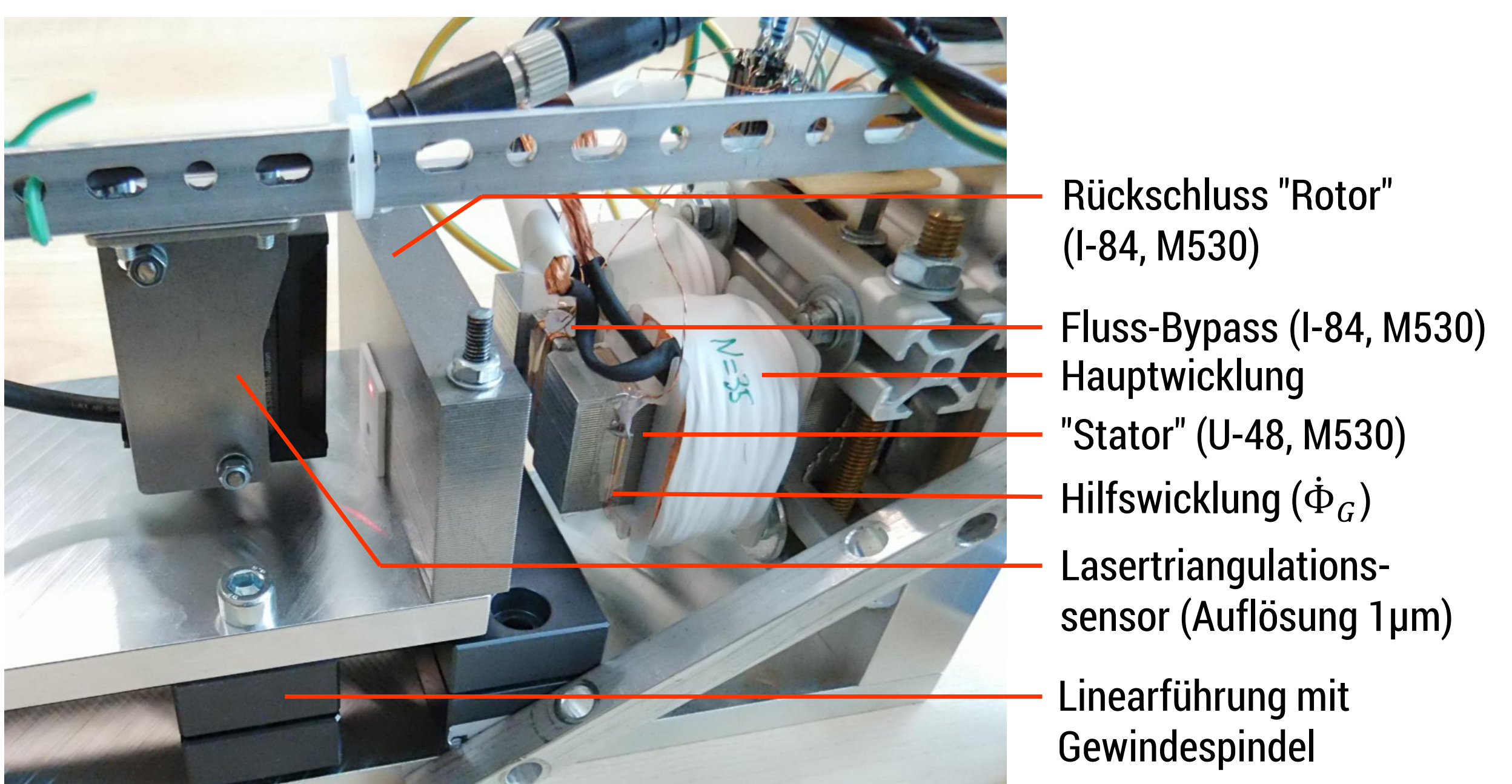




\section{Abtastrate und Grenzfrequenz}

Oszillogramm Messsignale (Verstärkerausgänge, vor ADC-Tiefpässen):

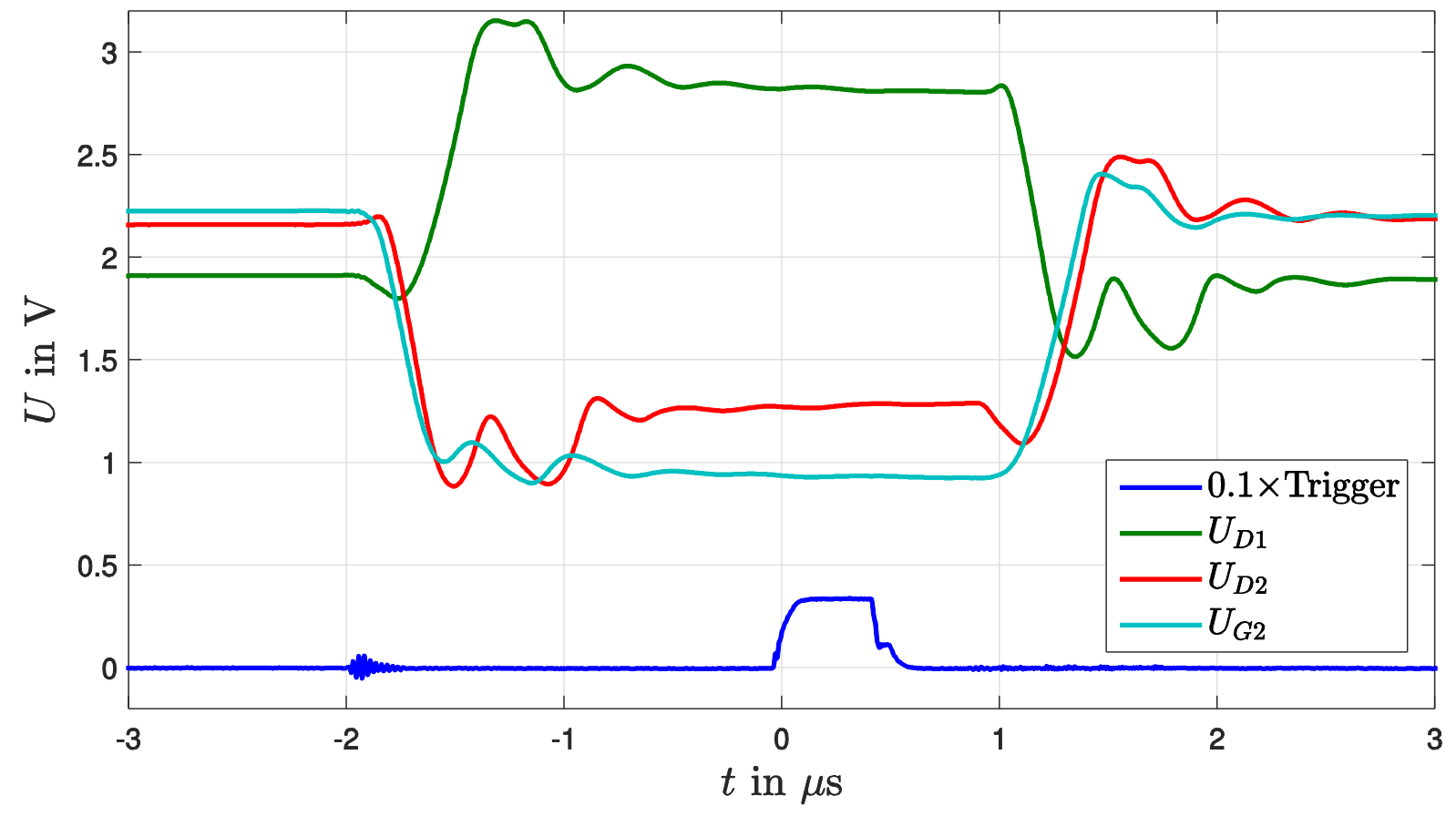

- Abtastung immer kurz nach Umschalten der Hauptwicklungsspannung möglich $\rightarrow$ hier: 1 Abtastung pro PWM-Periode: $\boldsymbol{f}_{\boldsymbol{A}}=\boldsymbol{f}_{\boldsymbol{S}}=\mathbf{5 0 \mathbf { k H z }}$

- Messgrenzfrequenz $f_{G}$ durch Grenzfrequenz der OPV-ADC-Anordnung sowie der Wicklungen bestimmt

$\rightarrow$ wegen transienter Messung gilt $f_{G} \gg f_{S}$, hier: $\boldsymbol{f}_{\boldsymbol{G}} \gg \mathbf{5 0} \mathbf{k H z}$ 


\section{Signal-Rauschabstand}

\section{Wichtige Rauschquellen}

- Widerstandsrauschen:

- im relevanten Frequenzintervall weiß, Rauschdichte [4]: $u_{N}=\sqrt{4 k T R} \approx$ $\frac{4 \mathrm{nV}}{\sqrt{\mathrm{Hz}}} \frac{\sqrt{R}}{\sqrt{\mathrm{k} \Omega}}$ bei Raumtemperatur

- Halbleiterrauschen:

- für OPVs derzeit möglich $u_{N} \lesssim \frac{1 \mathrm{nV}}{\sqrt{\mathrm{Hz}}}$ (für $f>10 \mathrm{kHz}$ ) [5], [6]

- für Vergleichsrechnungen: Low-Noise Hall-Elemente mit integriertem Chopper-Differenzverstärker: $S N R_{\text {Hall }} \lesssim 70 \mathrm{~dB}$ (bezogen auf Aussteuerbereich) [7]

\section{Allgemeines zum Signal}

- Änderung Signalstärke verbunden mit Änderung Parameter Leistungsteil (Blindleistung Motor, Verlustleistung Leistungshalbleiter, etc.)

- daher rel. enge Grenzen für Signalstärke

- hauptsächlich Signalquellimpedanz anpassbar (z.B. über Windungszahlen) 


\section{Signal-Rauschabstand}

- für Methoden nach Stand der Technik waren keine Angaben zum SNR auffindbar, daher optimistische Vergleichsrechnungen:

1. DDIE mit Shunt:

- Annahmen: Nennstrom $I=20 \mathrm{~A}$, Shunt: $R=50 \mathrm{~m} \Omega\left(P_{V}=20 \mathrm{~W} !\right)$

$\rightarrow$ Widerstandsrauschen vernachlässigbar

- Annahme: Stromänderung innerhalb einer PWM-Periode: $\Delta I=0,2 \mathrm{~A}_{\mathrm{PP}}$

- Aussteuerbereich: $U_{A B}=R \Delta I=10 \mathrm{mV}$

- Verstärkung mit OPV nötig, benötigte Bandbreite: Vielfaches der Schaltfrequenz $f_{S}=50 \mathrm{kHz}$, Annahme: $f_{B}=500 \mathrm{kHz}$

- Eingangs-Rauschspannung OPV: $U_{N} \approx \frac{1 \mathrm{nV}}{\sqrt{\mathrm{Hz}}} \sqrt{f_{B}}=0,71 \mu \mathrm{V}_{\mathrm{RMS}}$

- Rauschabstand: $\boldsymbol{S N R}<20 \log \frac{U_{A B}}{U_{N}} \approx \mathbf{8 3 d B}$

2. Streuflussmessung oder DDIE mit closed-loop-Stromwandler:

- Signale der Hallelemente enthalten Nutzsignal und deren Bandbreite kann nicht weiter reduziert werden, daher: $\boldsymbol{S N R}<S N R_{\text {Hall }} \approx \mathbf{7 0 d B}$ 


\section{Signal-Rauschabstand}

Hauptproblem von Shunts und Hallelementen:

- Fehlanpassung der Signalquellimpedanz an Verstärker: sehr kleine Spannung -> Spannungsrauschen Halbleiter dominiert

Fluss-Bypass-Verfahren:

- transformatorische Signalübertragung, hohe Signalleistung und Signalspannung, über Windungszahlen einstellbar

$\rightarrow$ gute Anpassung der Signalquelle

- höhere Windungszahl -> höherer SNR, jedoch höhere Zeitkonstante $L / R \rightarrow$ mögliche Abtastrate geringer

- Differenz-Wicklungsanordnung und volldifferenzielle Auswertung mit je zwei Instrumentenverstärkern für hohe Störunterdrückung

- keine numerische Schätzung der Zeitableitung nötig

-> Flussdifferenzierung über Induktionsgesetz 


\section{Signal-Rauschabstand}

\section{Abschätzung maximaler Rauschabstand Fluss-Bypass-Verfahren:}

- Wesentliche Rauschquelle: OPV

- äquiv. Eingangsrauschen: $e_{n}=\frac{1 \mathrm{nV}}{\sqrt{\mathrm{Hz}}}$, Ausgangsrauschen: $o_{n}=\frac{45 \mathrm{nV}}{\sqrt{\mathrm{Hz}}}$ [8]

- transiente Signalmessung $->$ hohe Signalbandbreite

-> hohe Rauschbandbreite: $f_{B}=1,67 \mathrm{MHz}$

- Verstärkungen: $V_{G}=3, V_{D}=6$-> Ausgangsrauschen dominiert

- differenzielle Auswertung

$\rightarrow$ Amplitude $\times 2$, Rauschen $\times \sqrt{2}$, da unkorreliert:

$$
U_{N, G} \approx U_{N, D} \approx \sqrt{2} o_{n} \sqrt{f_{B}} \approx 80 \mu \mathrm{V}_{\mathrm{RMS}}
$$

- Rohsignale:

- Aussteuerbereich $U_{A B}=8,192 \mathrm{~V}$

- Signal-Rauschabstand Rohsignale:

- $S N R=20 \log \frac{U_{A B}}{U_{N}} \lesssim \mathbf{1 0 0 d B}$ 


\section{Signal-Rauschabstand}

\section{Messung ( $\delta=1,5 \mathrm{~mm}, T=1 \mathrm{~s}$, alle Aussteuerbereiche $[-1,1])$ :}
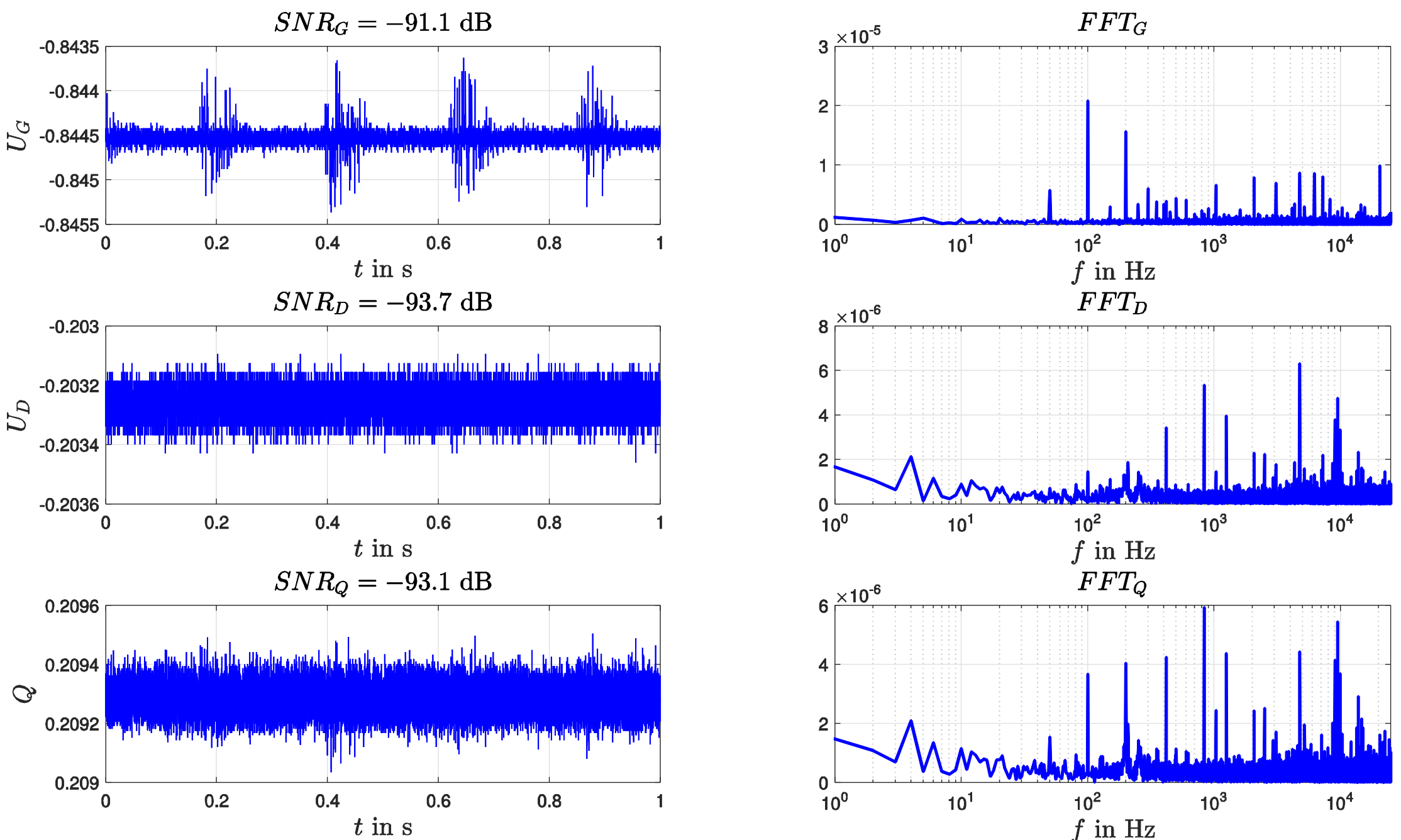

\section{- Rauschabstand Nutzsignal: $S N R_{Q}=93,1 \mathrm{~dB}$}


- Fluss-Bypass-Verfahren erreicht hohen SNR trotz hoher Bandbreite, höher als geschätzte Werte für andere Verfahren

- SNR von Nutzsignal $Q$ liegt typischerweise zwischen SNR der beiden Rohsignale $U_{G}$ und $U_{D}$

- gemessene Werte für SNR der Rohsignale ca. $4 \mathrm{~dB}$ bzw. $9 \mathrm{~dB}$ geringer als berechnete/simulierte Werte

- mögliche Ursachen: Gleichrichtung der Spannungsversorgung oder Netzeinkopplungen über Felder (FFT: deutliche Spitzen bei $100 \mathrm{~Hz}$ und Vielfachen)

- SNR von $U_{D}=U_{B}-U_{G}$ höher von $U_{G}$, möglicherweise wegen Subtraktion beider Wicklungsspg. (Gleichtaktunterdrückung) 


\section{Sättigungseinfluss}

Quasistatische Messempfindlichkeit (Invertierbarkeit von $\dot{\Phi}(\delta, \cdot))$ ):

$$
\varepsilon:=\frac{\partial U}{\partial \delta}=\frac{\partial^{2} \Phi}{\partial \delta \partial I} \dot{I}=\frac{\partial L_{d}}{\partial \delta} \dot{I}
$$

- quasistatische Messempfindlichkeit darf niemals null werden

- Sättigung kann jedoch dazu führen

-> Beispiel:

$$
\begin{aligned}
& \text { Sei } \Phi(I, \delta)=C \arctan \left(\frac{I}{k \delta}\right) \\
& \begin{aligned}
\Rightarrow \frac{\partial^{2} \Phi}{\partial \delta \partial I} & =C k \frac{I^{2}-k^{2} \delta^{2}}{\left(I^{2}+k^{2} \delta^{2}\right)^{2}} \\
& =0 f \ddot{u} r I= \pm k \delta \\
& >\text { kritischer Punkt }
\end{aligned}
\end{aligned}
$$

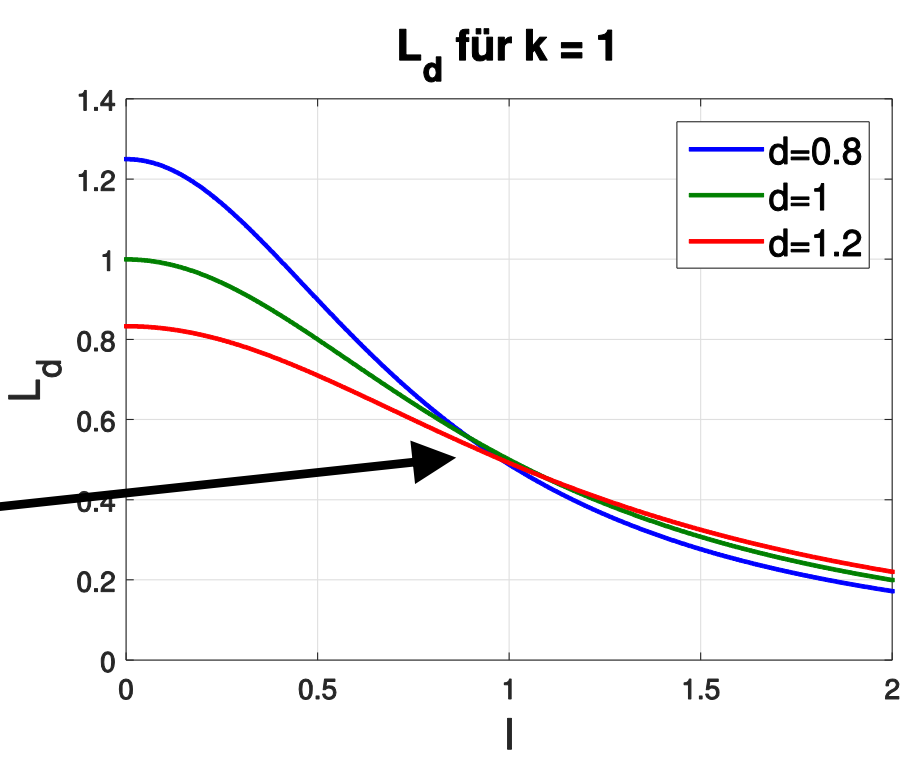




\section{Sättigungseinfluss}

\section{Simulation:}

- Schar statischer 2D-FEM-Berechnungen von $\Phi_{B}$ und $\Phi_{G}$ für äquidistant ansteigende Durchflutungen $\Theta$ sowie für verschiedene Luftspaltlängen $\delta$

- Approximation des Verlaufs von Nutzsignal $Q$ über Differenzenquotienten:

$$
Q(\Theta)=\frac{\frac{N_{B}}{N_{G}} \frac{\mathrm{d}}{\mathrm{d} t} \Phi_{B}-\frac{\mathrm{d}}{\mathrm{d} t} \Phi_{G}}{\frac{\mathrm{d}}{\mathrm{d} t} \Phi_{G}} \approx \frac{\frac{N_{B}}{N_{G}} \Delta \Phi_{B}-\Delta \Phi_{G}}{\Delta \Phi_{G}}
$$

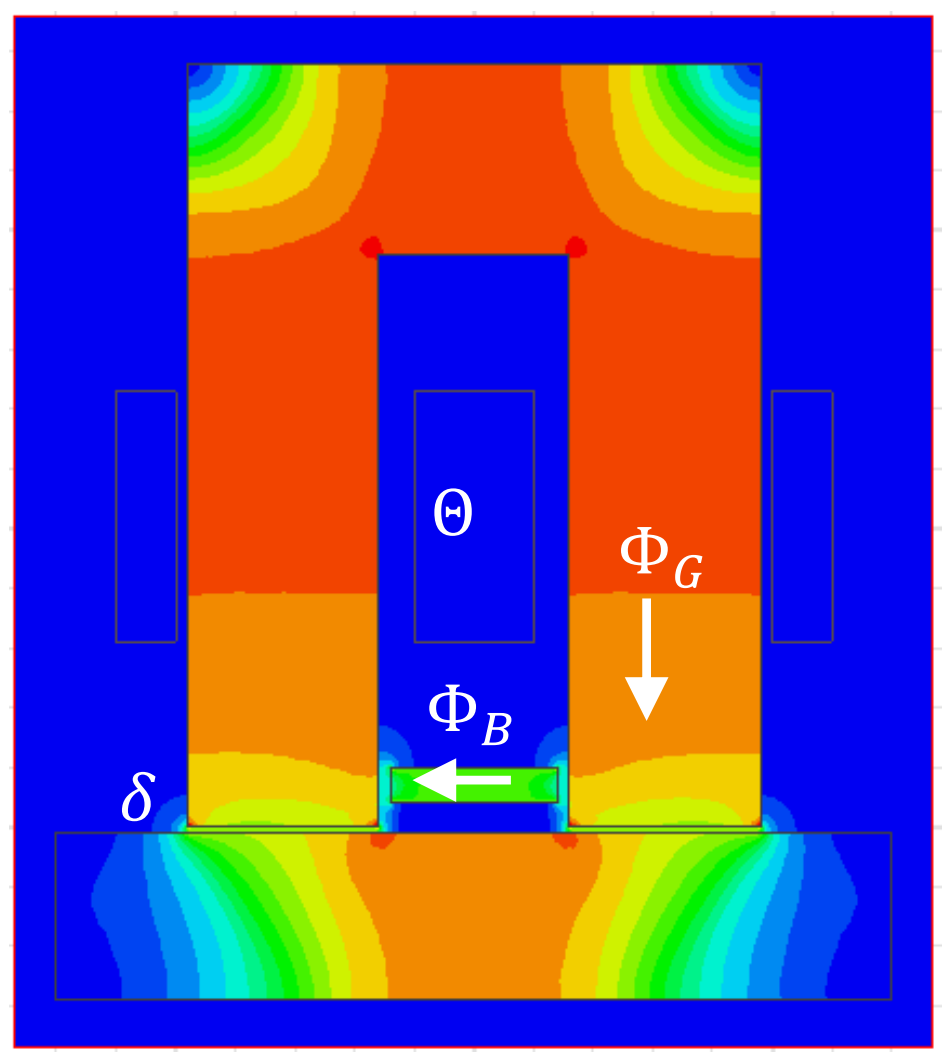

- zusätzlich: Vergleichsrechnung ohne FlussBypass-Kompensation:

$L_{d}\left(\Theta_{n}\right) \approx N_{G}^{3} \frac{\Delta \Phi_{G}}{\Delta \Theta}$ (nur mit diff. Induktivität - wie bei DDIE) 


\section{Sättigungseinfluss}

\section{Simulationsergebnisse:}
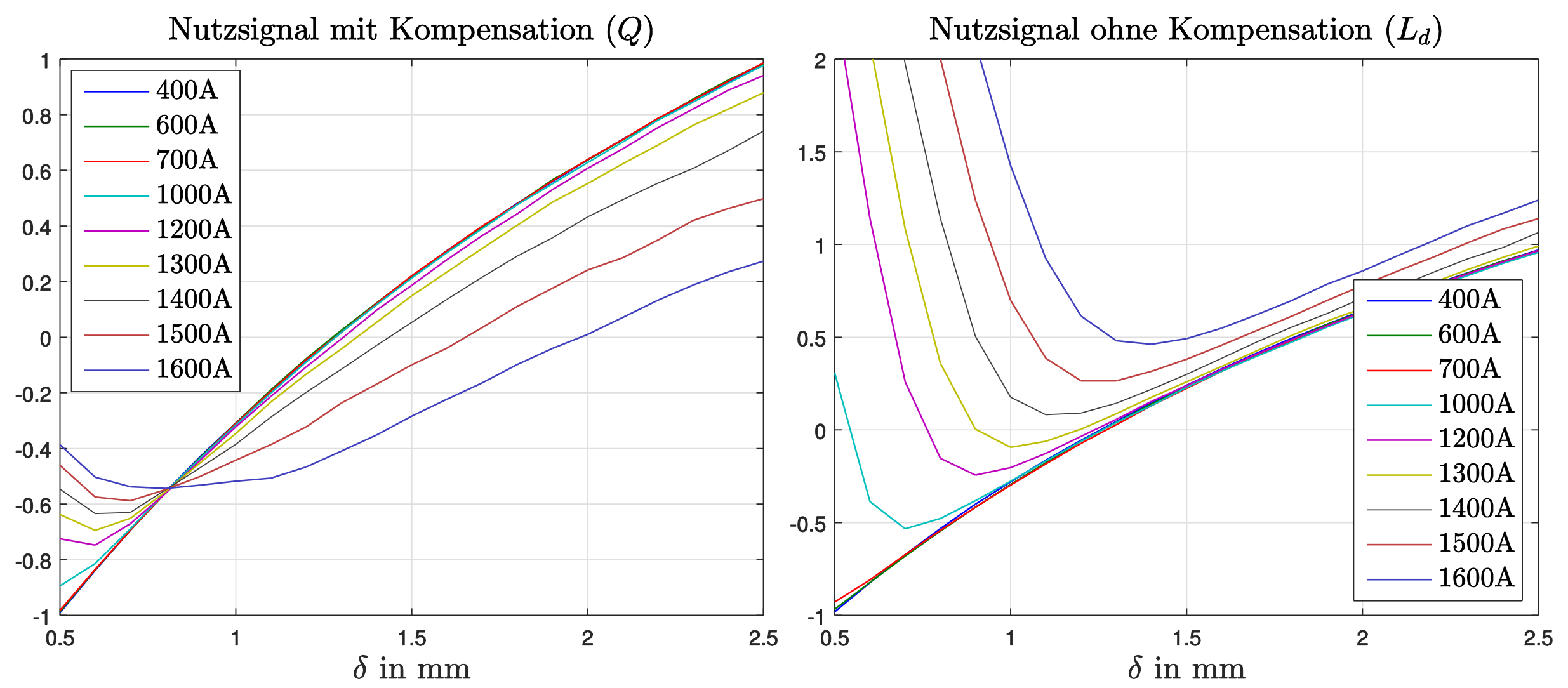

- für Vergleichbarkeit: Skalierung und Verschiebung beider NutzsignalKurvenscharen so, dass 400A-Kurven jeweils im Intervall [-1, 1] (vertikale Achse) 


\section{Sättigungseinfluss}

\section{Messergebnisse:}
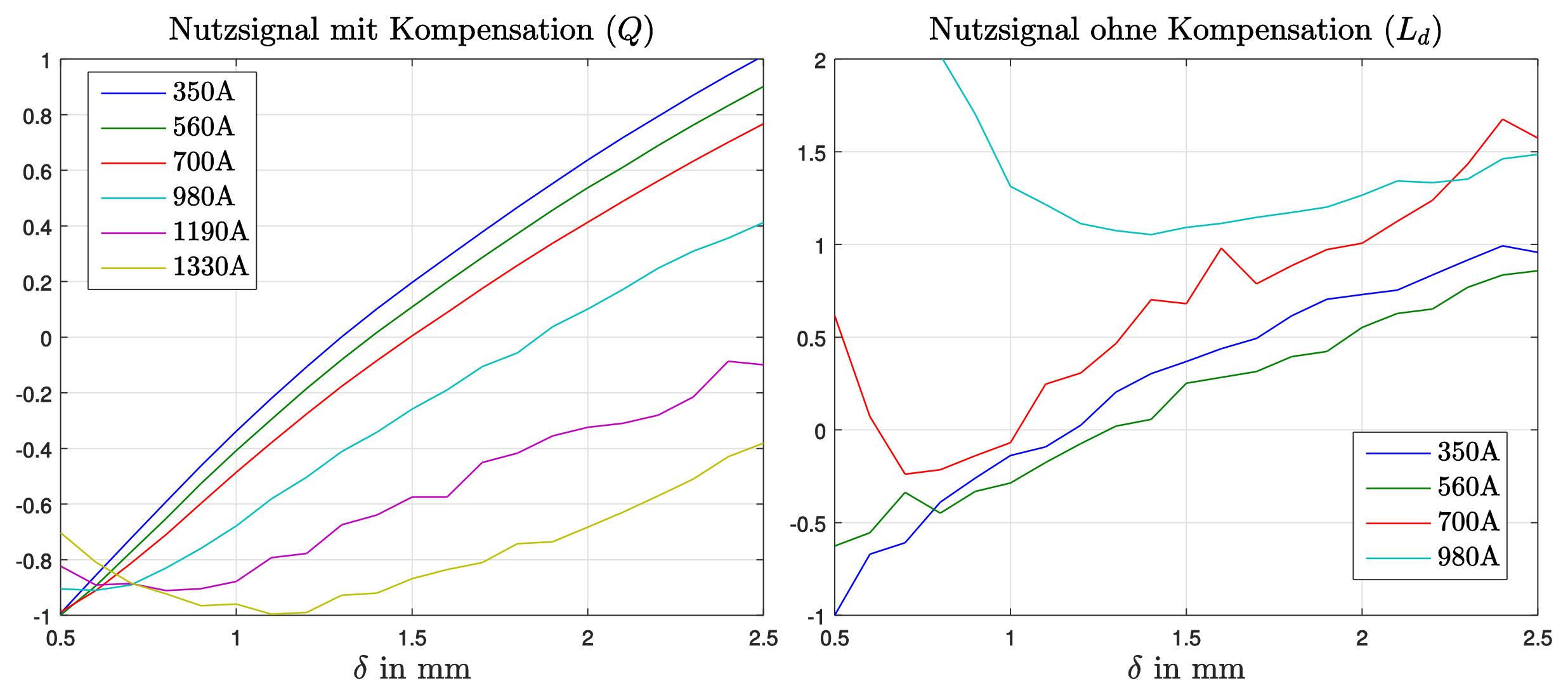

- bei höheren Stromstärken: Erwärmung führte zu Änderung Wicklungswiderstand -> Drift des Stroms (nur manuelle Nachregelung) -> Messwertschwankungen 
 \\ Sättigungseinfluss}

\section{FEM-Feldbilder bei $\delta=0,5 \mathrm{~mm}$ :}

kritischer Punkt mit Kompensation:
$\Theta=980 \mathrm{~A} \rightarrow \Phi_{G}=22.58 \mathrm{mVs}$

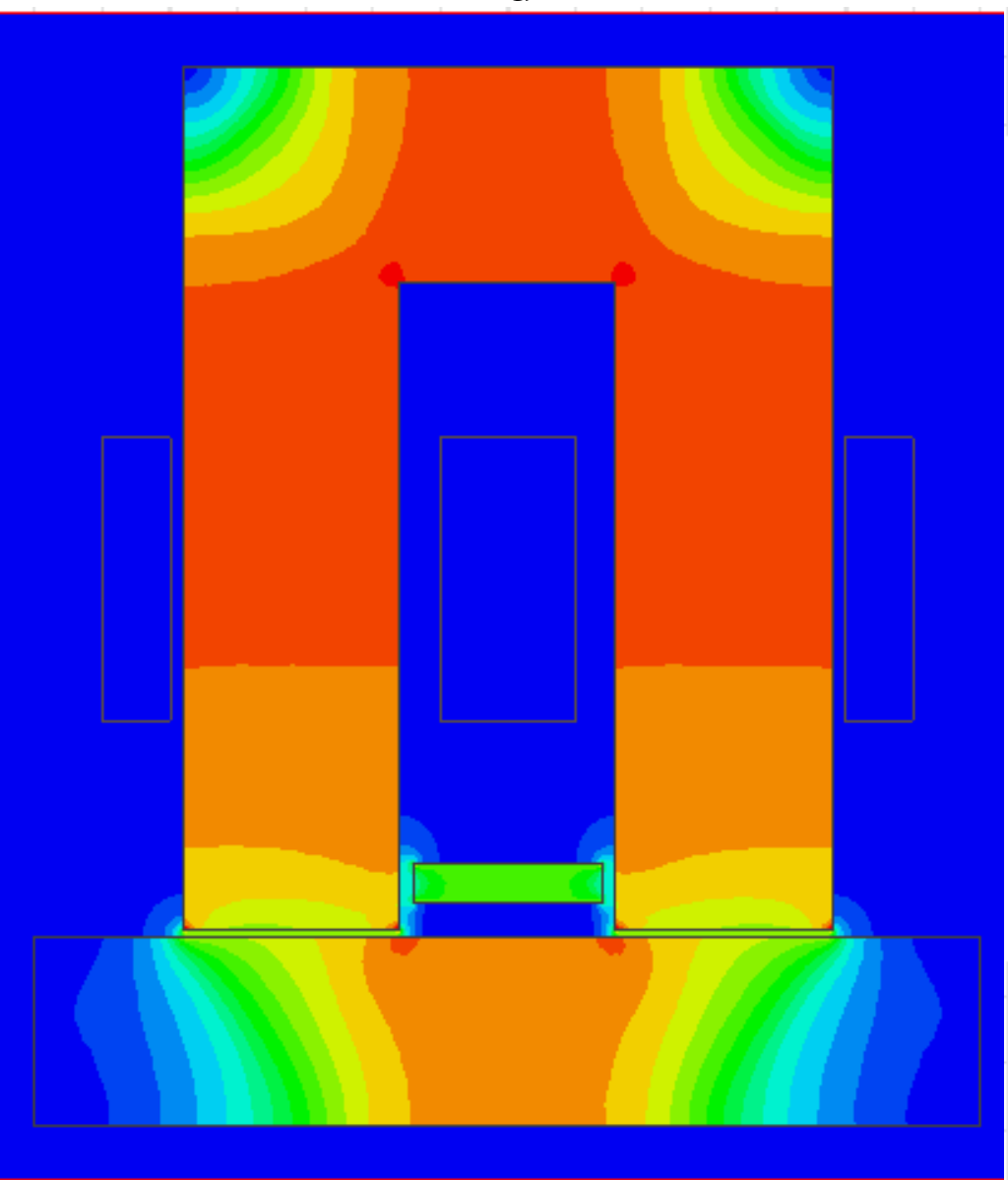

kritischer Punkt ohne Kompensation: $\Theta=630 \mathrm{~A} \rightarrow \Phi_{G}=15,56 \mathrm{mVs}$
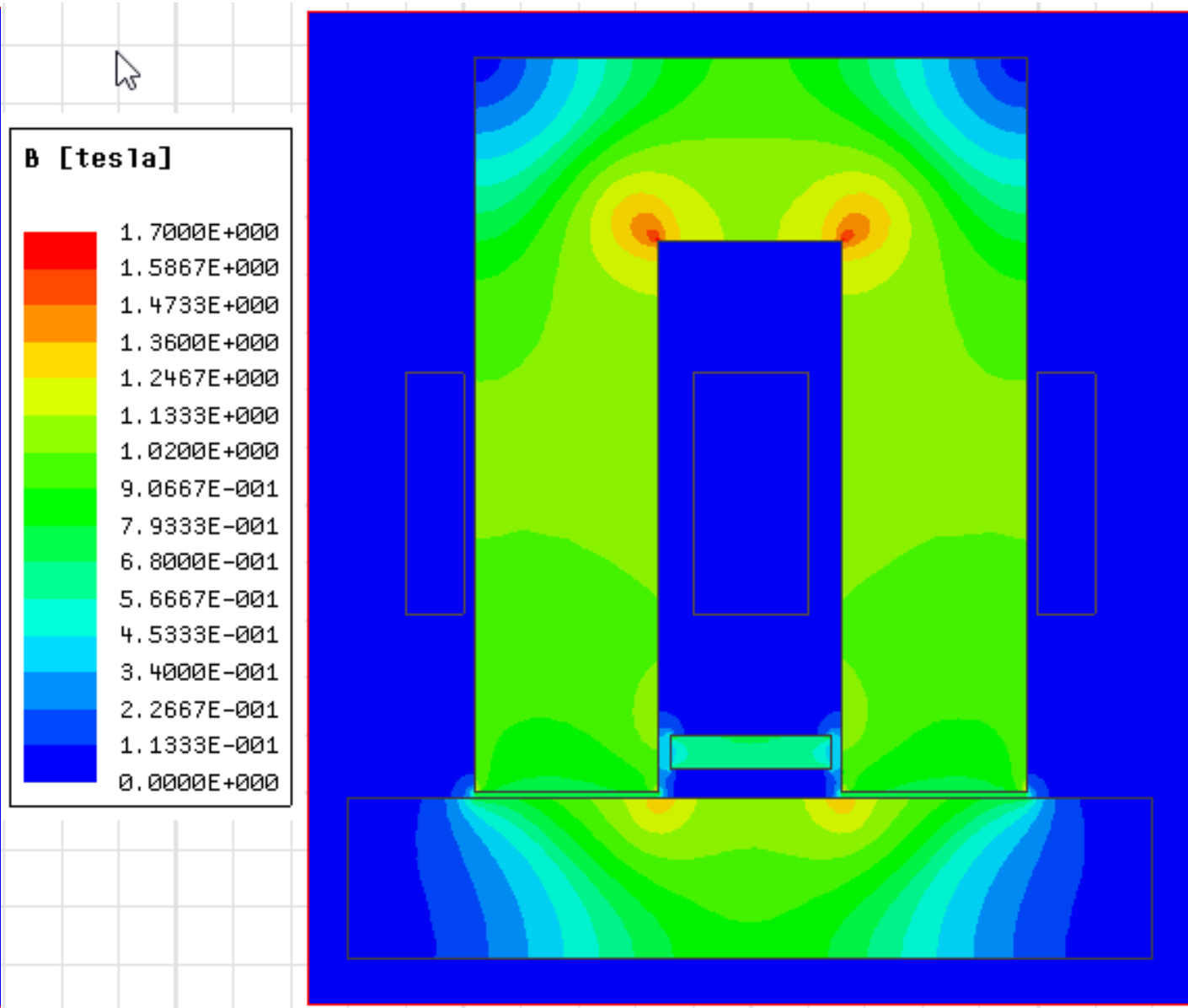


\section{Fazit Sättigungseinfluss}

- mit Fluss-Bypass-Kompensation deutlich mehr Sättigung tolerierbar als ohne:

- Durchflutung ca. +55\% (Messung)

- Fluss ca. $+\mathbf{4 5 \%}$ (FEM)

- Kraft ca. $+110 \%$ (FEM)

- gute Korrigierbarkeit der Kurvenschar mit Kenntnis des (langsam veränderlichen) Stroms

- Kurvenformen von Messung und Simulation stimmen näherungsweise überein

- jedoch in Messung scheinbar frühere Sättigung als in Simulation, mögliche Ursachen:

- Wirbelströme -> Frequenz der Spannung in Messung: $50 \mathrm{kHz}$ Rechteck, FEM: Materialkennlinie aus Datenblatt für $50 \mathrm{~Hz}$ Sinus

- FEM: 2D

- Materialbearbeitungseinflüsse \& Anisotropie 


\section{Fazit Fluss-Bypass-Verfahren}

\section{Ergebnisse Fluss-Bypass-Verfahren:}

- Abtastrate $\geq$ Schaltfrequenz, hier: $f_{A}=50 \mathrm{kHz}$

- Grenzfrequenz > Schaltfreq., hier: $f_{G}>50 \mathrm{kHz}$ (theoretisch)

- Signal-Rauschabstand hoch, hier: $S N R=93,1 \mathrm{~dB}$ (typisch)

- Kompensation Sättigungseinfluss über Fluss-Bypass möglich, da Sättigung innere Materialeigenschaft

-> ca. 55\% mehr Durchflutung zulässig

- Wirbelströme:

- äußern sich wie scheinbar erhöhte Sättigung

- können nur teilweise kompensiert werden, da keine innere Materialeigenschaft, sondern auch geometrieabhängig

Fazit: Gute Eignung für Magnetlagerungen und lagerlose Motoren 
- Weitere Untersuchungsmöglichkeiten:

- Einfluss Wirbelströme

- Einfluss Hysterese

- Optimierung Bypass-Geometrie, z.B. nicht rechteckiger Querschnittverlauf

- Differenzialanordnung zweier gegenüberliegender Messsysteme

- Vergleich Differenz-Wicklungen mit simplen Einzelwicklungen

- Erweiterung zu sensorlosem Magnetlager + Inbetriebnahme

- Erhöhung SNR:

- Beseitigung Störungen durch Netzteil/Netzeinkopplung

- Rauschreduktion Differenzverstärker (hochohmige interne Widerstände dominieren aktuell die Rauschdichte)

- Einsatz von Analog-Schaltern:

- Multiplexen mehrerer Kanäle (-> wirtschaftliche Lösung für Motor?)

- Chopperstabilisierung? 


\section{Quellen}

[1] T. Glück, W. Kemmetmüller, C. Tump, und A. Kugi, "A novel robust position estimator for self-sensing magnetic levitation systems based on least squares identification", Control Engineering Practice, vol. 19, no. 2, pp. 146-157, 2011.

[2] M. Richter, Hendrik Schaede, L. Quurck und S. Rinderknecht, "Untersuchung und Erhöhung der Praxistauglichkeit sensorloser Magnetlager nach dem "Direct Digital Inductance Estimation"- Konzept", Tagungsband 9. Workshop Magnetlagertechnik Zittau-Chemnitz, pp. 59-65, 2.-3.Sept. 2013.

[3] J. Rudolph, R. Werner: "Theoretical basics and closed loop control design of stray-flux-based measurement systems for magnetic bearings", The 15th International Symposium on Magnetic Bearings, Kitakyushu, Japan, 2016.

[4] D. Abbott, B. R. Davis, N. J. Phillips und K. Eshraghian, „Simple Derivation of the Thermal Noise Formula Using Window-Limited Fourier Transforms and Other Conundrums", IEEE Transactions on Education, vol. 39, no. 1, 1996.

[5] Analog Devices, Datenblatt LT1028, www. analog.com, Stand April 2018.

[6] Texas Instruments, Datenblatt OPA2211-EP, www.ti.com, Stand April 2018.

[7] Allegro MicroSystems, Datenblatt A1367, www.allegromicro.com, Stand April 2018.

[8] Analog Devices, Datenblatt AD8429, www. analog.com, Stand April 2018. 


\section{Danksagung}

Diese Arbeit entstand als Voruntersuchung für ein mögliches Fortsetzungsprojekt des aktuellen Projektes zum Thema „lagerloser reluktanter Rotations-Linear-Motor".

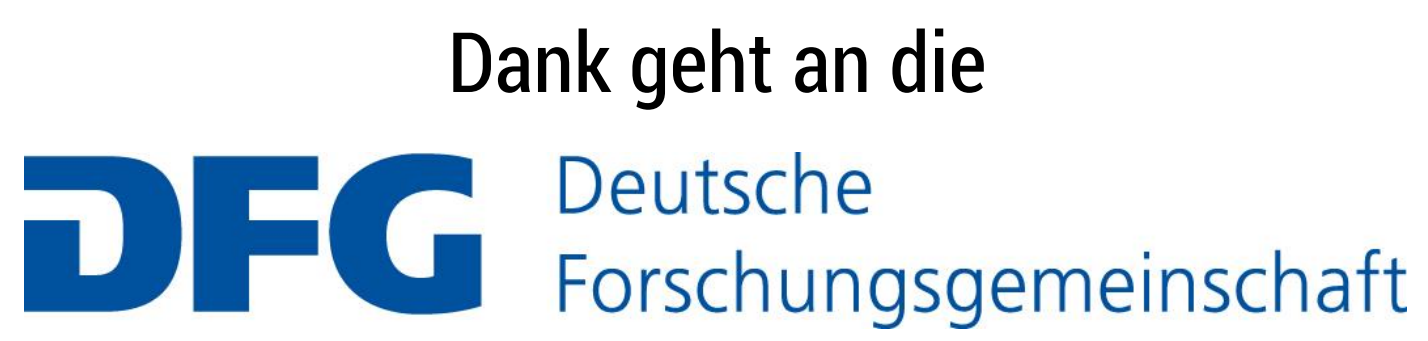

für die Förderung des aktuellen Projektes im Rahmen einer Sachbeihilfe (GZ: WE 4347/4-1). 